\title{
La science-fiction pour former des ingénieurs aux liens technique et société. L'imaginaire technologique comme élément de la culture technique
}

\author{
Using science fiction in engineers' education. Technological imagination
}

as an element of technical culture

\author{
Marianne Chouteau ${ }^{1}$, Céline Nguyen ${ }^{2}$ \\ ${ }^{1}$ Laboratoire S2HEP, Université de Lyon, INSA Lyon, France, marianne.chouteau@insa-lyon.fr \\ ${ }^{2}$ Laboratoire S2HEP, Université de Lyon, INSA Lyon, France, celine.nguyen@insa-lyon.fr
}

RÉSUMÉ. Raréfaction des ressources, changement climatique, artificialisation accentuée du monde, essor démographique, etc. sont autant de sujets où l'être humain et ses productions techniques sont impliqués. Ces grands enjeux contemporains nous invitent plus que jamais à ne pas séparer la technique de la société. Cela nous incite également à réfléchir aux leviers sur lesquels agir pour que les ingénieurs, producteurs de technique par excellence prennent conscience de leur pouvoir d'agir. Nous proposons pour ce faire de nous appuyer sur un élément de la culture technique, la science-fiction, comme voie d'entrée pour interroger le sens des objets conçus et de prendre en considération la part culturelle, souvent écartée, de la technique. Nous illustrerons notre propos par quelques exemples tirés de notre expérience en école d'ingénieurs.

ABSTRACT. Contemporary challenges such as resource scarcity, climate change and population growth require fresh thinking about the strong link between technology and society, especially for engineers who are responsible for technical production. In this context, we argue that technical culture is a valuable means to question the meaning of technical objects we design and use. This paper suggests that science fiction is a perfect way to gain access to this culture. Some examples from our experience in a French engineering school will illustrate this.

MOTS-CLÉS. Culture technique, imaginaire, ingénieur, innovation, science-fiction.

KEYWORDS. Technical culture, imaginary, engineer, innovation, science fiction.

\section{Introduction}

Le contexte général de ce début de $\mathrm{XXI}^{\mathrm{e}}$ siècle nous incite plus que jamais à nous intéresser et à nous interroger sur la technique. Raréfaction des ressources, réchauffement climatique, artificialisation accentuée du monde, développement du numérique, essor démographique, destruction de la biodiversité, etc. sont autant de sujets où l'être humain et la technique ${ }^{1}$ qu'il produit sont impliqués. Ces grands enjeux contemporains nous invitent à ne pas séparer la technique de la société. «L'enjeu est fondamental non seulement parce que les techniques accroissent la capacité d'action mais surtout parce qu'elles donnent la capacité de modifier la capacité d'action de l'homme » [VIN 88].

$\mathrm{Au}$ cœur de ce contexte sont situés les ingénieurs. Concepteurs, producteurs, avec d'autres, de technique, ils façonnent le monde avec leurs réalisations. De ce fait, il nous paraît naturel qu'ils puissent poser un regard critique tout à la fois sur leurs métiers et sur leurs productions. Il ne s'agit pas là de s'inscrire dans un discours à charge contre les ingénieurs. Mais plutôt de mettre en évidence, la nécessité qu'ils ont à comprendre ce qu'ils produisent, ici sur un plan symbolique, à travers la part d'humanité qu'il y a dans la technique. En d'autres termes, il s'agit de remettre la technique dans le cercle de la culture, d'en révéler la part culturelle. La technique en tant qu'activité humaine de production d'objets est en effet insérée dans un entrelacs de symboles, d'imaginaires, de croyances et de références culturelles. Pour ce faire, nous ferons un détour par la culture technique terreau

${ }^{1}$ Technique entendue dans le sens des procédés et des objets techniques résultant de ces procédés. 
indispensable à la compréhension de la technique et à sa production. Un certain nombre d'auteurs issus de disciplines différentes tels qu'André Leroi-Gourhan, Jocelyn De Noblet, Philippe Roqueplo, Jacques Perriault ou encore Bruno Jacomy ont milité pour le développement en France d'une « culture technique ». Leur argument principal était de dire : «Nous pensons donc qu'une culture technique est nécessaire parce qu'elle peut se définir comme la possession d'un minimum de connaissances et de savoir-faire permettant la réappropriation de notre environnement. Nous voulons dire qu'elle est une des conditions à partir desquelles il est possible de s'approprier la technique » [DEN 81].

Notre démarche s'inscrit dans le prolongement de ces initiatives militantes développées dans les années 1980 en France mais dans un contexte particulier, celui de la formation au métier d'ingénieurs, moment clé de la vie de l'ingénieur² ${ }^{2}$.

En effet, dans cette contribution, notre objectif sera de montrer que la science-fiction est un élément central de la culture technique dans la mesure où elle est un genre culturel particulièrement éclairant et apprécié des ingénieurs. La science-fiction permet de comprendre, à travers notamment les représentations, les imaginaires, les idéologies, etc., qu'elle véhicule ce qui inscrit socialement et symboliquement la technique, en tant qu'activité et l'objet technique en tant que résultat. Nous voulons montrer que les œuvres de science-fiction constituent un levier intéressant pour nourrir, travailler et rendre visible la culture technique de l'ingénieur et que ce faisant, elle a toute sa place dans une formation en école d'ingénieurs, qu'elle n'est pas simplement «une d'ouverture d'esprit» ou «une cerise sur le gâteau ».

Nous sommes donc bien conscientes que cet article porte sur les élèves-ingénieurs et la formation au métier d'ingénieur et non pas sur l'imaginaire des ingénieurs. Nous ne mesurons pas l'impact que ce genre de formation peut avoir sur leur pratique professionnelle et comment ils réinvestissent l'imaginaire lorsqu'ils sont en activité, à la sortie de l'école ou plus tard. En ce sens, cet article n'est pas le produit d'une enquête ou d'une évaluation dont on donnerait les résultats mais plutôt une proposition en acte qui tente de répondre aux défis qui leur sont aujourd'hui posés : concevoir éthiquement, s'inscrire dans des enjeux sociétaux prioritaires, être créatifs, etc., ce qui selon nous requiert une culture technique.

Aussi, dans cette contribution, après avoir défini la culture technique telle que nous l'entendons, dresserons-nous un rapide panorama sur la science-fiction pour en retracer rapidement l'histoire et les usages. Nous montrerons comment elle fait partie de la culture technique. Enfin, à travers des exemples de modules pédagogiques développés en écoles d'ingénieurs, nous mettrons en évidence comment la culture technique vient aux élèves-ingénieurs à travers la science-fiction.

\section{Qu'est-ce que la culture technique ?}

\subsection{Au nom de l'autonomie}

Très couramment, la culture technique a pour vocation de comprendre comment fonctionne un objet technique mais aussi d'en saisir la portée symbolique. André Leroi-Gourhan, dans la préface du Manifeste pour le développement de la culture technique témoigne : le rapport «fonction-forme» autrement appelé « design » est « une des voies privilégiées de la culture technique » [LER 81], faisant état d'une culture proche de la conception. L'anthropologue y incorpore, dans un deuxième mouvement, «l'esthétique » et les «institutions magico-religieuses » ce qui permet de comprendre la

\footnotetext{
${ }^{2}$ Nous sommes conscientes qu'il existe plusieurs sortes d'ingénieurs et que les profils et spécialités (R\&D, commercial, conception, etc.) des uns et des autres sont parfois très différents. La définition proposée par la commission des titres de l'ingénieur nous semble alors convenir : « le métier de l'ingénieur consiste à poser, étudier et résoudre de manière performante et innovante des problèmes souvent complexes de création, de conception, de réalisation, de mise en œuvre, de contrôle de produits et de systèmes ou de services éventuellement leur financement et leur commercialisation au sein d'une organisation compétitive. Il intègre les préoccupations de protection de l'homme, de la vie et de l'environnement et plus généralement du bien-être collectif » (Références et orientations de la CTI, 2015).
} 
valeur culturelle de l'objet technique par l'action qu'il rend possible. Ainsi, la culture entre dans l'objet technique qui à son tour produit du culturel.

Dès les années 1950, le philosophe des techniques Gilbert Simondon avait déjà dressé le constat selon lequel :

«La culture est déséquilibrée parce qu'elle reconnaît certains objets, comme l'objet esthétique, et leur accorde droit de cité dans le monde des significations, tandis qu'elle refoule d'autres objets, et en particulier les objets techniques, dans le monde sans structure de ce qui ne possède pas de significations, mais seulement un usage, une fonction utile. » [SIM 12]

Plus tard, la culture technique a fait l'objet d'une attention particulière dans les années 1980 notamment pour tenter de contrer l'ostracisation dont elle était victime, tout du moins en France, dans le champ de la culture légitime. Jocelyn de Noblet attire par exemple notre attention dans un manifeste pour la culture technique, au moment où l'on remet en cause un "progrès sans fin ", où l'on atteste de ses nuisances [DEN 81]. Il s'agit de prendre la mesure des systèmes techniques dans lesquels les objets techniques s'inscrivent, et à dépasser une simple familiarité avec les objets techniques pour acquérir des connaissances sur les objets techniques. Il la destine davantage aux citoyens : « la culture technique sera pour nous la pensée qui est à l'œuvre lorsqu'une technologie n'échappe pas à ses utilisateurs » [DEN 81]. Cette culture technique vise à sortir d'une double aliénation : celle de ne pas maitriser son environnement et de dépendre des organisations qui elles ont les compétences. Elle vise donc, pour cet auteur, à encourager l'autonomie.

Le propos de Philippe Roqueplo n'est pas éloigné :

« la culture technique consiste dans la possession des connaissances et savoir-faire susceptibles de fonder un minimum de maitrise personnelle sur notre environnement et de contrôle sur l'activité de ceux dont la compétence s'avère indispensable » [ROQ 83].

Pour ce faire, il y distingue trois définitions de la culture technique en fonction du point de vue que l'on adopte : «comment il a été fabriqué, comment on s'en sert, quand et pourquoi il a été inventé » [JAC 93]. Chacune d'entre elles vise à s'approprier l'objet technique dont il est question.

Yves Deforge nous invite quant à lui à penser que la culture technique

« exige la connaissance objective et opératoire du monde technique (et scientifique) mais aussi, au-delà, une aptitude à la pensée symbolique et universelle, c'est-à-dire proprement philosophique » [DEF 96].

Ainsi, l'auteur souhaite qu'interagissent des approches que l'on oppose souvent lorsqu'il est question de technique et que la part symbolique nourrisse l'action pour former un tout culturel :

« Ce que nous suggérons, c'est de mettre la technique et la science sous le contrôle d'une culture clairvoyante et agissante » [DEF 96].

En 1993, Bruno Jacomy définit plus précisément ce que peut être la culture technique de l'ingénieur, faisant de cette dernière un ensemble de connaissances liées à la technique et l'objet technique : connaissance de l'histoire des techniques et de l'industrie, de l'histoire de l'ingénieur, du processus d'innovation. Elle privilégie une approche culturelle de la technique : tout objet est représentatif d'une histoire, d'un système et s'inscrit dans un milieu socio-économique, politique particulier et vise à "mieux" innover, de développer des « techniques appropriées » [JAC 93]. 


\subsection{Pour une culture technique non segmentée}

Partant de ce terreau, nous avons proposé de définir une culture technique [CHO 17] qui s'adresse à une diversité d'acteurs dont les ingénieurs. Cette définition s'articule autour de trois niveaux d'analyse complémentaires.

Le premier niveau est celui qui concerne les calculs, matériaux, propriétés de l'objet. Ce sont les connaissances scientifiques et techniques qui définissent matériellement l'objet. C'est celui des connaissances relatives à la structure, à la composition de l'objet, aux fonctions; il est le niveau de la conception technique, celui des sciences de l'ingénieur classiquement définies. Ce niveau va de soi dans une école d'ingénieurs.

Le deuxième niveau est celui qui concerne l'usage de l'objet. Il permet de constater en quoi notre rapport à l'objet comporte une «épaisseur sociale » [JOU 00] lorsque nous l'utilisons. L'usage est fonction de nos projets, de notre mode de vie, de notre âge, de nos normes et valeurs, de notre activité, etc. Il modifie, accentue les pratiques et permet de mettre au jour les logiques d'usage [PER 98], les stratégies et tactiques [DEC 90] [JAU 11]. Ce niveau permet ainsi de rendre compte de l'activité de l'utilisateur, de tout ce qui se joue dans son rapport à l'objet. Par exemple, c'est ici que l'on peut rendre compte de l'adoption très rapide du mobile par les parents divorcés et leurs enfants, leur permettant de développer des conversations en toute autonomie et liberté.

Le troisième niveau concerne le sens plus global de l'objet. Ce sont les connaissances symboliques, politiques, idéologiques, etc. qui permettent de comprendre l'objet et les changements qu'il engendre à une échelle plus large. Il inscrit l'objet dans les grands enjeux contemporains : la réduction des déchets, l'accès à la santé, à l'éducation... Ce dernier niveau est de nature politique car il invite à penser l'orientation que la technique donne à la société qu'elle contribue à façonner. Il se place à une échelle citoyenne, permettant notamment de débattre des technologies.

La culture technique telle que nous l'entendons suppose que ces trois niveaux ne soient pas segmentés et surtout qu'ils ne soient pas travaillés en silo. Un concepteur (ingénieur ou non) peut aussi réfléchir au sens de l'objet du point de vue du citoyen ou de l'usager. Un usager peut devenir concepteur et s'interroger sur le sens politique de sa pratique. Cette conception des choses ne va pas forcément de soi dans une école d'ingénieurs. En effet, si le premier niveau est facilement accessible pour les élèves-ingénieurs, le deuxième et le troisième le sont moins. Notre propos est donc de faire en sorte que les élèves-ingénieurs (et les ingénieurs par la suite) aient conscience de ces trois niveaux afin qu'ils mettent en place une réflexion globale les articulant. Autrement dit, qu'ils soient en mesure, de penser ensemble, les fonctions et la matérialité de l'objet, ses usages et ses implications plus politiques et idéologiques.

\section{Science-fiction, technique et récit : des rapprochements fertiles}

La science-fiction véhicule des représentations, porte des promesses ou des appréhensions que l'on pose sur la technique et en cela, elle participe à la symbolisation de la technique. Elle constitue alors l'un des ingrédients de la «culture technique » telle que nous venons de la définir en se situant aux 3 niveaux. Elle revêt d'autres atouts : la science-fiction est populaire chez les élèves-ingénieurs. Elle fait partie de leur culture. S'ils ne sont pas tous de grands lecteurs - on compte toutefois parmi eux quelques lecteurs assidus - ils sont dans leur grande majorité spectateurs (cinéma ou séries) [CHO 15a]. De plus, la science-fiction - quel que le soit le support par lequel elle est diffusée - offre la possibilité de faire un détour, de ne pas entrer directement dans les questionnements mais de les aborder par d'autres voies. Elle permet d'investir la culture technique avec/par les élèves-ingénieurs. 


\subsection{La science-fiction, un genre socio-technique}

Nous ne dresserons pas ici l'histoire complète et exhaustive du genre mais il convient toutefois de rappeler qu'une de ses fonctions, à ses débuts dans les années 1920, fut selon son inventeur Hugo Gernsback celle d'un outil de vulgarisation, un genre fait pour susciter des vocations chez les scientifiques et les ingénieurs [CON 12]. Il fit le pari qu'en donnant à voir des mondes technologiques possibles, la science-fiction pouvait provoquer l'envie. De nombreux auteurs se sont penchés sur les apports de la science-fiction. L'un des plus souvent évoqués est celui d'exprimer des désirs et des craintes : désir d'une société meilleure, crainte d'un monde dystopique. Le caractère fictionnel des récits et des personnages constitue même un atout :

«Les personnages romanesques saisissent mieux la vérité d'une période que les personnages réels appréhendés par les sociologues ou les historiens » [TOF 17]

Pierre Musso définit la science-fiction comme «un genre littéraire contemporain de la révolution industrielle, qui cherche à concevoir un monde différent, voire à prédire l'avenir technique et social. Née dans une période de foi dans le progrès, la science-fiction est désormais marquée par des interrogations sur les conséquences néfastes de certaines innovations » [MUS 05]. En écho à Gilbert Hottois [HOT 00], il avance que la science-fiction contemporaine aurait en effet perdu son « idéalisme » issu de la modernité pour « explorer la société humaine » et s'inscrire dans le post-modernisme.

Dans la première partie de Fabriquer le futur, Pierre Musso propose de passer par l'imaginaire pour montrer que l'innovation est aussi une «construction historique et sociale » [MUS 05] et pas seulement une production qui va de soi, sans socle culturel. L'auteur montre très bien comment l'imaginaire est central dans le processus de conception (phase centrale du processus d'innovation) via la pluralité des imaginaires portés par les acteurs du processus : concepteurs mais aussi usagers, grandes organisations, littérateurs, publicitaires, tendanceurs. Il montre comment ces médiations portent des utopies, des idéologies ou s'appuient sur des grands mythes et à ce titre, il accorde alors à la science-fiction un statut à part comme vectrice d'utopies sociales.

La science-fiction est également un marqueur culturel. En effet, selon les pays, elle ne joue pas le même rôle. Si la science est perçue comme un mal fascinant en France, la perspective est plus critique en Grande-Bretagne lorsqu'il s'agit de penser son usage. Aux Etats-Unis, la littérature est plus optimiste. Cela s'explique par le fait que les auteurs viennent davantage des sphères littéraires alors qu'ailleurs ils naviguent davantage dans des sphères scientifiques [KLE 16], donc plus empreints d'autocritique. Par ailleurs, les imaginaires évoluent dans le temps. A propos des techno-imaginaires, Georges Balandier [CHA 94] observe une mutation: les «utopies techno-messianiques » sont progressivement remplacées par des utopies «techno-catastrophiques» signe de temps bien plus critiques et de cette perte d'idéalisme.

Le genre littéraire ou cinématographique «science-fiction » a ceci de particulier qu'il fait de la science et de la technique l'un de ses ingrédients principaux. Genre d'exploration, d'impulsion, de frontière [CON 12], il est l'un des vecteurs les plus pertinents nous permettant d'explorer, par la fiction, notre lien à la technique. En effet, les imaginaires présents dans les récits de science-fiction sont largement portés par les objets techniques qui peuplent les univers proposés : navette spatiale, habitat souterrain, voiture volante, sabre laser, robots humanoïdes, humains modifiés ou augmentés... Non seulement ceux-ci sont les marqueurs de ce genre mais ils font aussi avancer le récit en tant qu'accessoires voire personnages principaux ou secondaires.

Ce faisant, la technique présente dans la science-fiction «est toujours un mixte de tekhné et de logos » [MUS 13] ; c'est-à-dire qu'elle est à la fois le résultat d'un acte technique, d'une fabrication et qu'elle engendre de la connaissance et un discours, une parole. Cette parole est celle de l'objet technique en lui-même qui n'est jamais muet mais aussi celle de l'auteur et du public qui la reçoit. 


\subsection{La science-fiction : un genre particulier au service de la culture technique}

En quoi ce genre constitue-t-il alors un levier de développement d'une culture technique ? Pour tenter de répondre à cette question, nous avons identifié trois fonctions complémentaires que les récits de science-fiction peuvent jouer dans le cadre d'une culture technique à développer et ce, aux trois niveaux proposés.

La première fonction est celle d'impulser et d'entourer la technique d'un imaginaire significatif. La science-fiction, riche en imaginaire technologique peut en effet fonctionner comme un réservoir d'idées, de suggestions, d'envies. Si les auteurs de science-fiction puisent dans l'actualité et la « réalité » scientifique pour construire leurs récits, à l'inverse, le genre permet aux concepteurs d'enclencher un processus d'innovation, soit en lançant un défi qu'une nouvelle technologie pourrait relever (une nouvelle façon de se déplacer, de s'alimenter...) soit en suggérant plus ou moins directement une solution technique à un problème identifié. S'il va de soi que la science-fiction ne fournit jamais de solution clé en mains, elle peut malgré tout permettre de formuler des vœux, d'être une source d'inspiration [RUM 16-17] ou de lancer des pistes de travail.

L'Agence spatiale européenne a par exemple produit un rapport en 2002 intitulé Les nouvelles technologies et la science-fiction ${ }^{3}$ fruit d'une collaboration entre chercheurs, ingénieurs et auteurs qui ont dressé une liste d'idées à creuser, recherché dans les œuvres de fictions des inventions et innovations qui pourraient être développées et repéré les idées viables. Les combinaisons des vols spatiaux ont été notamment analysées et plus particulièrement les tenues de combinaison vinyl ou encore les vêtements récupérateurs d'eau dans Dune. Bien plus tôt, et même si l'alchimie ne relève pas de la science-fiction, cette dernière a constitué un moteur de connaissances sur le métal durant le Moyen-Age [GAR 05]. Plus près de nous et pour Gérard Klein, le transhumanisme s'inspire clairement de la science-fiction. L'un de ses plus fervents défendeurs, ingénieur chez Google et fondateur de l'université de la Singularité, Ray Kurzweil annonce par exemple que l'homme fusionnera avec l'intelligence artificielle en 2045 ou qu'il disparaitra dépassé par les machines ${ }^{4}$.

Le domaine informatique - et ce n'est pas étranger au transhumanisme et à la convergence NBIC sur laquelle il s'appuie - semble marqué de l'empreinte d'une fiction qui remonte loin dans le temps, celle des « créatures artificielles »:

«Scientifiques et ingénieurs de ce domaine [informatique, ndlr] rêvent, depuis plus de 50 ans, de construire un « robot intelligent » qui serait à «l'image de l'homme ». Ici le futur, rejoint les mythes passés, et la science se conjugue étroitement avec l'imaginaire » [BRE 13].

De son côté, le sociologue Patrice Flichy [FLI 89] a bien montré que le désir d'universalité a été fondateur en matière de télécommunications. Offrir des moyens de communication à n'importe qui, n'importe où (soit la révolution proposée par les téléphones portables) nous permet par exemple de faire un lien avec le mythe de Babel ; de même, les inventeurs d'appareils enregistreurs ont été animés par la volonté de reproduire en série des images et du son [FLI 89], une quête d'immortalité en quelque sorte. Enfin, l'auteur a également très bien montré comment l'histoire d'Internet est faite d'utopies et d'idéologies présentes dans l'esprit des fondateurs comme dans les discours des relais médiatiques [FLI 01].

Dans cette perspective, les travaux de Pierre Musso suivent une posture intéressante car il lui parâtt nécessaire de garder à l'esprit que l'imaginaire est un ingrédient d'une innovation réussie dans le sens où celui des concepteurs doit coïncider avec celui des usagers. L'auteur parle alors de « coélaboration des représentations » soit la «convergence et partage de références » [MUS 05]. Son propos vise à dépasser une vision manichéenne des choses : d'un côté, le rejet de l'imaginaire par les uns, parce que

\footnotetext{
${ }^{3}$ Voir http://cds.cern.ch/record/600385/files/cer-002359434.pdf

${ }^{4}$ Lire par exemple : https://www.01 net.com/actualites/ray-kurzweil-espere-la-fin-de-1-humanite-pour-dans-douze-ans-1125964.html 
considéré comme irrationnel donc inexistant, et de l'autre une adhésion voire une fascination particulièrement visible dans «l'imagerie produite par des campagnes médiatiques standardisées » [MUS 05], imagerie exploitée pour faciliter l'adoption d'un produit ou service ou pour initier un projet de recherche. Ainsi, il met en lumière le versant culturel de toute innovation : les ingénieurs et techniciens baignent dans des univers technologiques fictionnels plus ou moins standardisés qui, irriguent leurs désirs. Ces univers sont parfois identiques à ceux des usagers ou s'opposent, se complètent, ce qui montre que dans l'objet comme dans notre lien à lui se jouent des mécanismes complexes. Thomas Michaud [MIC 14] a quant à lui bien montré l'importance de l'inconscient prophétique très présent chez certains informaticiens du service R\&D d'Orange comme celle de son exploitation managériale ou dans le registre du marketing.

La seconde fonction est de nature plus réflexive: elle est celle qui permet l'analyse des représentations et in fine, la compréhension de notre rapport à la technique. Les œuvres de fiction sont particulièrement pertinentes pour mettre en scène des objets techniques, des usages, des applications qui relèvent d'une intention politique, de valeurs, de choix de société. Ce faisant, cela nous invite, que nous soyons ingénieurs, hommes politiques, usagers et/ou citoyens, à repérer les promesses et les peurs liées au devenir de la société et à la technique qui en fait partie :

«Les spectateurs sont aussi des citoyens et ils ont besoin de la science-fiction pour se fixer une vision du futur collectivement partagée » [GUE 14]

La force de ce genre est donc de nous inciter à nous projeter, à mobiliser notre imaginaire pour penser les contours de la société souhaitée ou redoutée de sorte à prendre des chemins pertinents et éviter les pièges. En créant, les auteurs de science-fiction s'inspirent nécessairement de la société contemporaine pour en exagérer certains traits. Ils donnent ainsi à réfléchir à partir des explorations socio-économiques, des dysfonctionnements réels ou sous-jacents.

Ici, ce n'est pas la prédiction qui est intéressante ou recherchée mais la compréhension des trajectoires de la société pour faire des propositions. En ce sens, la prospective reste la discipline qui exploite le plus le genre car «le prospectiviste est légitimement tenté de rechercher des pistes dans ce continent de représentations imaginaires de l'avenir que constitue la littérature de science-fiction ». [KLE 16]

Par ailleurs, l'imaginaire technologique central dans le genre met en lumière les mythes et les failles de l'Humanité. Si comme nous l'avons dit les mythes peuvent inspirer la création technique, ils permettent aussi de souligner nos failles, à l'image du mythe de Prométhée qui, d'une part permet de comprendre en quoi la technique donne du pouvoir à l'homme mais d'autre part nous invite à prendre conscience de la démesure technologique. Et c'est l'interprétation du mythe de Prométhée qui change également avec les époques et notre relation à la technique comme le souligne Dominique Lecourt [LEC 96]. L'imaginaire prégnant des TIC renvoie très largement à l'imperfection humaine au profit d'une intelligence artificielle et au transhumanisme dont nous avons parlé plus haut.

Brigitte Munier montre que la science-fiction a questionné à travers le temps l'image d'un homme créateur de créatures artificielles.

« (...) La science s'empara du Golem-robot: des œuvres sans cesse plus nombreuses questionnent toujours aujourd'hui la relation entre le démiurge humain et la créature humanoïde et surpuissante qu'il a fabriquée. » [MUN 13].

Elle cite à titre d'exemple : Metropolis, Blade Runner, les nouvelles d'Asimov, etc. En regardant par exemple un épisode de la série suédoise Real Humans nous voyons une société possible pourvue de hubots (robots humanoïdes) dont l'objectif est de nous assister, nous soigner... laissant entrevoir toutes les faiblesses physiques et psychologiques humaines (lassitude, erreur, maladie, ...). Mais nous les 
voyons également se substituer à l'être humain en raison de leur perfection supposée ce qui n'est pas sans poser de questions.

La dernière fonction est complémentaire de la fonction réflexive. Elle est de nature cognitive et lie plus fortement le genre au statut fictionnel et aux propriétés narratives. En effet, si la science-fiction est propice à la réflexion c'est parce qu'elle permet une expérience de la pensée, une immersion. Souvent dystopique, elle place au cœur de son intrigue des problèmes politiques, sanitaires ou économiques largement traités; les objets techniques en sont d'ailleurs régulièrement les marqueurs ou révélateurs. C'est ainsi que les spectateurs ou lecteurs peuvent en retour imaginer des solutions (techniques ou non) car poussés par le schéma narratif qui les conduit à la résolution de problème. Le caractère fictionnel souvent très éloigné du réel laisse alors une plus grande liberté dans l'expérimentation [RUM 16] : «A sa manière, en jouant sur les ressorts de l'imaginaire, la science-fiction offre des ressources cognitives de nature à stimuler les formes d'espérance et à leur ouvrir des espaces expérimentaux » affirme dans ce sens le politiste [RUM 16-17]. La fiction est parfois plus à même de nous permettre d'avoir une vision interne des choses, un accès, une médiation plus évidente :

«Grâce à l'expérience sensible qu'elle met en œuvre, la fiction littéraire propose justement une vision du dedans qui s'impose comme supérieure à la vision du dehors, prisonnière de la pensée conceptuelle » [TOF 17].

\section{La science-fiction et l’imaginaire au cœur de la formation}

La science-fiction dans les enseignements de sciences humaines et sociales en école d'ingénieurs est-elle vraiment mobilisée ? Une rapide consultation du site Internet de plusieurs d'entre elles ne permet pas vraiment de répondre à cette question car les cursus ne sont pas toujours détaillés. Il y a fort à parier que les enseignements artistiques tournés vers la littérature ou le cinéma puisent dans ce vivier. Pour autant, un enseignement entièrement dédié à ce genre et au service du développement d'une culture technique telle que nous l'avons définie ne semble pas fréquent. Notre expérience à l'INSA Lyon $^{5}$, support de notre réflexion/proposition s'inscrit d'ailleurs dans cette «configuration »: si la culture technique guide notre contribution aux enseignements, la science-fiction est seulement l'un des moyens d'y parvenir ${ }^{6}$. L'enjeu est alors de taille : il s'agit de faire en sorte qu'une culture personnelle, libre et relevant du divertissement devienne alors un support de formation, un objet de connaissances et de réflexion intégrable dans une future pratique professionnelle. Cela ne va pas toujours de soi car cela oblige à rapprocher des cultures que l'on oppose souvent, la culture littéraire et la culture scientifique [SNO 59] ; les étudiants comme les institutions ne sont pas toujours prêts à sauter le pas.

\subsection{Explorer les représentations de la science-fiction}

Cette approche est plutôt celle d'une initiation à la question des représentations, question approfondie plus tard. Il s'agit en effet de s'appuyer sur une fiction d'anticipation pour donner accès aux représentations véhiculées par la science-fiction et d'en analyser les enjeux. Ici, la fonction de projection des récits de science-fiction dans des mondes possibles est utilisée. Quels sont les mondes futurs envisagés? Qu'est-ce que cela nous dit sur notre monde présent ? Pourquoi ? Quels questionnements en retirer ? Le premier exemple est celui de la lecture en classe du prologue intitulé «Un monde parfait » de l'ouvrage critique d'Eric Sadin La vie algorithmique (2015). Cet essai débute en effet par le récit de la journée type d'un homme, dont on s'aperçoit qu'elle est parfaite, sans accroc car réglée par les algorithmes: la température de son lit, la nature du thé sont fonction de ses

\footnotetext{
${ }^{5}$ L'objectif de cet article n'est pas de dresser un panorama exhaustif de toutes les expériences pédagogiques incorporant l'analyse des œuvres de science-fiction dans une formation au métier d'ingénieurs ou dans un cursus universitaire scientifique. D'autres expériences existent, sans doute plus disruptives, et témoignent de la volonté de s'adapter aux nouveaux publics, de privilégier des logiques d'immersion, de ludification pour lesquelles l'imaginaire et en particulier la science-fiction peuvent jouer un rôle central. Mais nous avons pris le parti, ici de traiter de ce que nous avons expérimenté.

${ }^{6}$ Afin de faire accéder les futurs ingénieurs à la compréhension des logiques d'usagers, nous les invitons par exemple à mener des enquêtes de terrain, à questionner les pratiques, etc. 
constantes, les trajets sont optimisés, etc. L'exagération subtile de ce texte permet alors de questionner ce monde possible. Lors de la séance, chaque étudiant lit à voix haute à tour de rôle, quelques lignes de cet ouvrage. Ainsi, au fil de la lecture, chacun s'implique, s'imagine et se projette dans le monde décrit. A-t-on envie de vivre dans ce monde commode, pratique, efficace et parfait? La discussion collective se construit autour des enjeux liés au numérique : celui de la mesure permanente et de la liberté, du respect de la vie privée, de la fainéantise, de l'immortalité.

Le second exemple concerne la robotique : il s'appuie sur l'analyse d'une séquence du film $E x$ machina (Garland, 2014) ${ }^{7}$ montrant l'interaction particulièrement oppressante d'une intelligence artificielle avec un informaticien censé la tester. Au cours de cette séquence, le jeune informaticien est censé déterminer, à l'aide d'une espèce de test de Turing, si l'intelligence artificielle a une conscience de soi. La séance vise non seulement à comprendre qu'à travers les choix du réalisateur, il existe des représentations fortes qui alimentent un imaginaire collectif autour des robots humanoïdes : c'est une femme, elle est faite de métal, des fluides bleus la traversent, elle a une plastique agréable, des traits du visage avenants... Mais ce travail permet aussi de s'interroger sur un enjeu central en science-fiction : celui du dépassement de l'intelligence humaine par celle des machines. La scène montre une illustration concrète des doutes émis par les êtres humains. Elle met également en évidence la puissance de ces machines.

L'utilisation de la science-fiction possède une autre vertu qui se situe dans le prolongement de celles évoquées plus avant : en prenant conscience de l'imaginaire technologique des œuvres de fiction, les élèves ingénieurs sont plus disposés à comprendre les projections et représentations des usagers, de mieux cerner et comprendre le rapport à la technique tant d'un point de vue individuel que collectif. Car en effet, si l'élève-ingénieur joue le jeu jusqu'au bout, il pourra avoir accès à ses propres peurs et aux espoirs qu'il met sur la technique, mais il pourra aussi effectuer le même travail réflexif en se projetant sur ceux des potentiels utilisateurs. Enfin, il pourra aussi s'interroger sur quels bouleversements seront engendrés si telle ou telle technique est déployée. C'est sans doute à ce niveau qu'il aura accès à l'aspect politique de la technique. Par exemple, il pourra se poser la question du libre arbitre : qu'est-ce que cela veut dire sur notre liberté de penser ? Ou encore la question de la sélection des individus : est-on aujourd'hui dans une société eugéniste? Qu'est-ce que cela signifie sur notre façon d'accepter ou non le handicap?

L'objectif pédagogique de ces trois exemples est alors d'ouvrir la discussion entre les étudiants et de faire émerger chez eux des questionnements tout en leur permettant de s'interroger sur les valeurs qu'ils accolent à la technique.

\subsection{La science-fiction pour construire une démarche éthique}

Depuis longtemps, la science-fiction a été pensée comme un terreau particulièrement fertile pour penser l'éthique. On le sait les récits qu'elle produit sont porteurs d'imaginaires apocalyptiques ou prophétiques qui incitent, si on en a conscience, à une réflexion éthique. A l'instar de Dominique Vinck, nous pensons que «l'étude de l'imaginaire a un rôle à jouer dans la réflexion éthique » [VIN 88].

Aussi, la première sensibilisation expliquée ci-dessus est approfondie plus tard dans les études et notamment dans un département Biosciences par un module centré sur l'éthique. L'objectif est de s'appuyer sur l'utilisation de la fiction et plus spécialement de la fiction d'anticipation pour penser l'éthique et mettre en place une véritable démarche.

Il s'agit de sortir d'une vision manichéenne de la technique ou de celle, restrictive, d'une éthique qui dirait le bien et le mal. A contrario, se servir de l'univers de la science-fiction leur permet de réinterroger «l'imagination éthique » [PIE 12] en créant de nouveaux mondes dans lesquels se projeter.

\footnotetext{
${ }^{7}$ Les œuvres sur lesquelles nous travaillons (en particulier cinématographiques ou « télévisées ») sont celles qui restent accessibles au public visé tout en mettant en scène des problématiques profondes, non traitées de manière superficielle et d'actualité. 
En effet, les propriétés narratives de la science-fiction permettent aux élèves-ingénieurs de prendre conscience des idéologies associées à certaines techniques [MIC 14]. Par exemple, en analysant certains films de science-fiction où il est question de biotechnologies ou de techniques médicales, les élèvesingénieurs s'interrogent tout à la fois sur le mythe de la santé parfaite [SFE 95] que sur l'imperfection et la vulnérabilité de l'homme [BRE 13].

Aussi, le module d'enseignement se divise en deux temps. Le premier est consacré à l'analyse de fictions cinématographiques (films ou séries) pour la plupart issues de la science-fiction. Les films analysés sont choisis par les étudiants en fonction de leur sensibilité et cela va de Bienvenue à Gattaca (Niccol, 1997) à Westworld (2016-2018) en passant par Terminator, ou la saga Alien. Ce premier temps de travail permet l'analyse des représentations des sciences et des techniques véhiculées par ce type de fictions. Il est poursuivi par un deuxième consacré à la construction d'une démarche éthique. Par exemple, alors que les élèves-ingénieurs analysent la façon dont les robots sont représentés dans ces différents récits cinématographiques, ils s'interrogent par exemple sur la nature de notre humanité. Qu'est-ce qui nous distingue d'un Terminator, robot tout à la fois destructeur et protecteur, qu'est-ce qui peut a contrario nous rapprocher des robots de Westworld, ces humanoïdes capables de conscience et de mémoire ? Chemin faisant l'analyse de ces représentations peut leur permettre par exemple de s'interroger sur l'altérité, sur cet autre qui nous permet par ricochet de mieux nous connaître. Cette étape d'analyse n'est pas anodine car elle pose les jalons d'une réflexion éthique individuelle et collective : qu'est-ce qui semble acceptable ? Quels sont les éléments que l'on peut discuter, négocier et ceux qui ne relèvent d'aucune négociation possible ? Ces éléments sont ensuite remobilisés dans un travail complémentaire ou lorsque, pour approfondir leur réflexion, les élèves-ingénieurs conçoivent des dispositifs comme une matrice éthique - sur le modèle de celle de Ben Mepham ${ }^{8}$, organisent des conférences de citoyens, ou rédigent des textes argumentatifs de type avis du Conseil Consultatif National d'Ethique sur une question donnée. La construction de cette démarche éthique se poursuit enfin par leur mise en représentation lors d'une journée où confrontés aux autres étudiants, aux enseignants et à des conférenciers, les élèves-ingénieurs présentent le résultat de leurs réflexions éthiques.

Ce travail permet aux futurs ingénieurs d'avoir un regard rétrospectif : que redoutait-on ou dans quelles promesses se projetait-on dans les années 1980 - ou prospectif - qu'est-ce que nous promettent aujourd'hui les sciences et les techniques et quelles sont les appréhensions de notre temps et pour l'avenir.

Cette approche analytique se poursuit par une étape que l'on peut qualifier de plus «productive». En effet, nous demandons aux élèves-ingénieurs de produire eux-mêmes un récit soit sous forme de nouvelles fictionnelles, soit sous forme théâtralisée. Cette étape est donc plus personnelle et permet d'approfondir le travail préalablement construit autour des représentations. Aussi, la réflexion éthique ne se construit-elle pas ex-nihilo.

En effet, en écrivant, les élèves-ingénieurs prennent conscience de leur propre positionnement éthique et défendent les valeurs qui leur sont importantes. L'écriture et qui plus est la mise en récit permettent de structurer la pensée, de la mettre en ordre et de rendre cohérents des éléments épars. Aussi, lorsque l'on place l'élève-ingénieur en position d'écriture, la fonction d'expérimentation éthique du récit s'exprime en plein. L'écriture fictionnelle inspirée de l'analyse de ces récits de science-fiction permet à l'élève-ingénieur de se forger un positionnement éthique [KEM 97].

«L'anticipation littéraire permet en effet d'écrire ce que l'on veut - que l'on désire ou que l'on redoute, au fond c'est la même chose - à propos de ce qui peut advenir » [KLE 16].

\footnotetext{
${ }^{8}$ La matrice éthique est un outil d'aide à la réflexion éthique. En 1996, elle a été conçue par Ben Mepham, biologiste et éthicien. Elle a entre autres pour vertu de prendre en compte tous les pluralismes (valeurs et acteurs) et de ne pas limiter l'éthique à un positionnement surplombant. Au contraire, elle permet d'intégrer la réflexion éthique tout au long du processus d'innovation. 
Par exemple, lors de l'atelier d'écriture qui s'est tenu en 2018, un élève-ingénieur a mis en scène un échange épistolaire amoureux entre une intelligence artificielle affranchie et un jeune homme, enfermé dans un hôpital psychiatrique car amoureux d'une entité virtuelle. A travers cet écrit, l'étudiant a composé un monde où l'intelligence artificielle est plus humaine que les humains dans sa capacité à ressentir l'amour et l'empathie. Aussi a t-il pu, entre autres, s'interroger sur sa façon personnelle de définir ce qu'est l'humanité et comment se définissent les frontières entre l'homme et la machine.

$\mathrm{Au}$ final, on le voit aisément, l'utilisation de l'imaginaire à travers les œuvres de science-fiction comme terreau pour concevoir, penser, voire même pour imaginer l'éthique est tout à fait probante. La fiction permet de dépasser, de s'affranchir du réel et de se lancer dans une discussion décomplexée où on ne s'inquiète pas de savoir si telle ou telle chose est possible, plausible ou vraisemblable.

\subsection{Perspectives : récolter et s'appuyer sur les imaginaires de la science-fiction pour innover}

Lors d'une émission sur France Inter le 10 août 2018, Alain Damasio, l'auteur de science-fiction affirmait que ses ouvrages ne sont pas là seulement pour décrire des sociétés dystopiques et alerter l'opinion publique sur d'éventuelles dérives mais qu'ils étaient aussi là pour ouvrir les possibles. Nous l'avons dit plus haut, tout objet technique porte en lui sa fonctionnalité et sa «fictionnalité » [MUS 14] et vouloir séparer ces deux éléments amènerait à une mauvaise compréhension de ce qu'est cet objet. Cette fonction «d'aide à la conception» pourrait être exploitée dans un module de formation à l'innovation en année de spécialisation. Ceci aurait entre autres pour dessein de proposer tout au long du processus de conception une posture réflexive sur la technique. Cette proposition permettrait alors de ne pas penser la technique seulement à travers ses impacts sur la société, une fois que l'objet est pensé, mais au contraire de l'intégrer dès la conception.

L'objectif de cette démarche serait d'inciter les élèves-ingénieurs à puiser dans le terreau fertile de l'imaginaire en s'inspirant des objets ou univers qu'ils ont pu voir représentés ou décrits dans la science-fiction.

Pour ce faire, un module de formation pourrait être mis en place en exploitant précisément l'imaginaire dans le processus de conception. En effet, en s'appuyant en partie sur la méthodologie développée par la Chaire MODIM $^{9}$, il serait intéressant que les élèves-ingénieurs prennent conscience des imaginaires en lien avec la science-fiction et s'en servent pour innover. Dans leur ouvrage Innover avec et par les imaginaires rédigé dans le cadre de cette chaire, Stéphanie Coiffier, Pierre Musso et Jean-François Lucas développent une méthodologie de cartographies pour modéliser les imaginaires afin de favoriser l'innovation. Il s'agit alors d'identifier une «rationalité de l'imaginaire » [MUS 05] formalisable sous forme de grilles d'analyse. Aussi, en nous appuyant sur les différentes étapes qu'ils déploient - établissement d'un corpus de documents ordonné spatialement et temporellement, retours sur les expériences d'usage vécues, mises en scène théâtralisées - développer un procédé similaire pour inciter les élèves-ingénieurs à se lancer dans un processus d'innovation. Ils pourraient concevoir une cartographie des imaginaires de l'objet technique sur lequel ils travaillent, au moment de la phase d'idéation avec la consigne de s'orienter plus spécialement sur les récits de science-fiction et de dresser une carte heuristique à partir des œuvres, univers et objets de domaines techniques variés. Il s'agit là non seulement d'accéder aux représentations partagées, mais aussi de s'inspirer, de trouver des sources de motivation pour améliorer un objet ou en imaginer un nouveau.

Lors de la phase plus tardive d'utilisation de méthodologies de créativité, les élèves-ingénieurs pourraient intégrer des éléments issus de la science-fiction au moyen d'un outil comme la matrice de découverte comme des sabres laser, vaisseaux spatiaux, voitures volantes, etc. et les croiser avec les éléments ou fonctions d'un objet à inventer ou améliorer. Ce passage par la science-fiction

\footnotetext{
${ }^{9}$ La Chaire MODIM (Modélisation des imaginaires, innovation et création) vise en effet à développer une recherche fondamentale et appliquée autour de l'expérimentation et de la formation sur les imaginaires des acteurs de l'innovation. Rassemblant chercheurs et industriels, elle s'est tenue entre 2010 et 2015. 
encouragerait le décentrement, l'audace imaginative, le détour, avant de revenir sur une phase plus réaliste.

\section{Conclusion}

Pour conclure, nous pouvons dire que si le fait d'utiliser les œuvres de science-fiction pour accéder aux projections et croyances autour de la technique et des innovations n'est pas nouveau, s'en servir comme l'un des accès à une culture technique «englobante » et non segmentée capable d'aider les futurs ingénieurs à œuvrer dans une démocratie technique l'est peut-être un peu plus.

On pourra reconnaitre que cette approche n'est pas réservée aux ingénieurs et techniciens tant nous sommes tous concernés par le développement technologique. La science-fiction, grâce à son art du détour, peut également constituer un bon support pour lancer des débats de citoyens dans différents dispositifs de médiation. On pourra aussi reprocher à cette proposition d'être difficilement transférable dans le monde des entreprises et cela est sans doute vrai, d'autant que la science-fiction y est parfois convoquée pour de «mauvaises raisons » [MIC 14]. Néanmoins, nous espérons avoir, par ce biais, convaincu certains futurs ingénieurs que leur action fait société et qu'elle est proprement culturelle. Pour finir, il nous semble que la science-fiction, a trois atouts majeurs.

Elle est un genre fictionnel qui, parce qu'il amplifie, caricature parfois, donne accès très facilement à la signification de la technique à travers les représentations et en particulier à celles des objets techniques qui la «peuplent». Sa puissance narrative en fait un levier médiatique fort : si « L'utopie de la science-fiction est un de ces micro-discours qui médiatise les progrès techniques dans de nombreux secteurs » [MIC 14] elle constitue également une voie d'entrée par laquelle entamer un questionnement sur la place de la technique et trouver des éléments de réponse. Il s'agirait alors de faire de ces atouts communicationnels une force.

Si l'on se place ensuite sur le terrain de l'innovation, on peut également supposer que le caractère souvent décalé des œuvres de science-fiction ouvre la voie à une prise de conscience voire à des innovations qui s'affranchissent du déterminisme [RUM 16]. La science-fiction nous offre un prétexte riche pour travailler la technique car elle met en évidence la complexité des mondes et incite à y réfléchir.

Enfin, le travail critique et réflexif rendu possible par l'intermédiaire de la science-fiction pourrait permettre aux futurs ingénieurs d'avoir accès au sens et aux significations de la technique, de prendre place dans la démocratie technique et de ne pas s'enfermer dans une vision manichéenne technophobe ou techno-euphorique, souvent paralysante.

\section{Bibliographie}

[BRE 13] BRETON P., « Pourquoi l'ordinateur n'a pas de parole ?», dans MUNIER B. (dir), Technocorps. La sociologie du corps à l'épreuve des nouvelles technologies, ED. François Bourin, Paris, 2013.

[CHA 94] CHANIAL P., B ALANDIER G., « Entretien avec Georges Balandier ». Quaderni, n²3, Printemps in Science(s) de la communication. p. 119-132., 1994.

[CHO 11] Chouteau M., NGuYen C. (dir.), Mises en récit de la technique. Regards croisés, Editions des Archives Contemporaines, Paris, 2011.

[CHO 15a] Chouteau M., Faucheux M., NGuyen C., «Les élèves ingénieurs à l'épreuve de la fiction. Quelles relations entretiennent-ils avec les mondes scientifiques et techniques fictionnels ? » dans Les Enjeux de l'Information et de la Communication, ${ }^{\circ} 16 / 3 \mathrm{~A}$, p. 69-82, 2015. Consulté le vendredi 20 juillet 2018, [en ligne] URL : http://lesenjeux.u-grenoble3.fr/2015-supplementA/22-Chouteau-Faucheux-Nguyen/index.html.

[CHO 15b] Chouteau M., Escudié M.-P., Forest J., Nguyen C., «L'ingénieur, au cour de la démocratie technique », dans Lamard P., et Lequin Y., (dir.), Eléments de démocratie technique, Presses de l'UTBM, Belfort, 2015. 
[CHO 15c] Chouteau M., Escudie M.-P., Forest J., NGuyen C., «La technique est-elle condamnée à entrer par effraction dans notre culture », Phronesis, vol. 4, n 2, p.5-16, 2015. Consulté le mardi 11 septembre 2018, [en ligne] URL : https://www.erudit.org/fr/revues/phro/2015-v4-n2-phro02105/1033446ar/.

[CHO 17] ChOuteau M., Forest, J., NGUYen C., «Quand la culture d'innovation fait écran à la culture technique », Technologie et Innovation, $\mathrm{n}^{\circ} 17-4$, septembre, 2017. Consulté le mardi 11 septembre 2018, [en ligne] URL :https://www.openscience.fr/Quand-la-culture-d-innovation-fait-ecran-a-la-culture-technique

[CHO 18] Chouteau M., Forest J., NGuyen C, «Concevoir en donnant du sens à l'innovation : l'approche P.S.I », dans J. DidiER, N. BonNARDEL \& D. LeUBA (Dir), Didactique de la conception. Presses de l'UTBM, Belfort, 2018.

[CON 12] CONVERT B, Demailly L., «Effets collatéraux de la création littéraire. L'exemple de la science-fiction., Sociologie, Opus 21, p. 111-133, 2012.

[DEC 90] De CerTeau M., L'invention du quotidien. 1. Arts de faire, Paris, Gallimard, 1990.

[DEN 81] De NOBLET J., « Culture technique et changement de société », Culture et Technique, n6, p. 11-47, 1981.

[FLI 89] FLICHY P., «L’imaginaire collectif des ingénieurs : le cas des machines à communiquer », Réseaux, n³6, p.5370, 1989.

[FLI 01] FLICHY P., L'imaginaire d'Internet, La Découverte, Paris, 2001.

[GAR 05] GARÇON A.-F., «Les techniques et l'imaginaire. Une question incontournable pour l'historien », Hypothèses, p. 221-228, 2005.

[GUE 14] GUERMONPREZ P., Les drônes débarquent, Ed. FYP, 2014.

[JAC 93] JACOMY B, «Culture technique de l'ingénieur, Techniques de l'ingénieur », 17 p., 1993 ; Consulté le mardi 11 septembre 2018, [en ligne] URL : https://www-techniques-ingenieur-fr.docelec.insalyon.fr/res/pdf/encyclopedia/tiagc-t40-version1.pdf

[JAU 11] JAUREGUIBERRY F.ET PROULX S., Usages et enjeux des technologies de la communication, Eres, Toulouse, 2011

[JOU 00] JOUET J., « Retour critique sur la sociologie des usages », Réseaux, n¹00, p. 487-521.

[KEM 97] KEMP P., L’irremplaçable. Une éthique technologique, Ed. Cerf, Paris, 1997.

[KLE 16] KLEIN G., «L’invention de l'avenir : prospective et science-fiction », Futuribles, n4113, p. 29-52, 2016.

[LEC 96] LeCourT D. Prométhée, Faust, Frankenstein : Fondements imaginaires de l'éthique, Biblio Essai, Paris, 1996.

[MIC 14] MiCHAUD T, «La dimension imaginaire de l'innovation. L'influence de la science-fiction sur la construction du cyberespace », Innovation, n44, p. 213-233, 2014.

[MUS 05] Musso P. Ponthou L., SeUILlEt E., Fabriquer le futur. L'imaginaire au service de l'innovation, Le village mondial, Paris, 2005.

[MUS 13] MUSSO P., «Le technocorps, symbole de la société technicienne », dans MUNIER B. (dir), Technocorps. La sociologie du corps à l'épreuve des nouvelles technologies, Ed. François Bourin, Paris, 2013.

[MUS 14] MUSSO P., COIFFIER S., LUCAS J-F., Innover avec et par les imaginaires, Ed. Manucius, Paris, 2014.

[MUN 13] MUNIER B., «Le Golem ou les vertiges d'un homme fabriqué » dans MUNIER B. (dir), Technocorps. La sociologie du corps à l'épreuve des nouvelles technologies, Ed. François Bourin, Paris, 2013.

[PER 98] Perriault J., La logique de l'usage. Essai sur les machines à communiquer, L'Harmattan, Paris, 1998.

[PIE 12] PIERron P., Les puissances de l'imagination. Essai sur la fonction éthique de l'imagination, Ed. Cerf, Paris, 2012.

[ROQ 83] RoQUEPLO P., Penser la technique. Pour une démocratie concrète, Ed. Le Seuil, Paris, 1983.

[RUM 16] RUMPALA Y., « Tester le futur », Futuribles, n413, p.53-72, 2016.

[RUM 16-17] RUMPALA Y. «Entre imaginaire écotechnique et orientations utopiques ». La Science-fiction comme espace et modalité de reconstruction utopique du devenir planétaire, Quaderni, 97-117, hiver 2016-2017.

[SFE 95] SFEZ L., La santé parfaite. Critique d'une nouvelle utopie, Ed. Le Seuil, Paris, 1995.

[SIM 12] SimONDON G., Du mode d'existence des objets techniques, Ed. Aubier, Paris, 2012.

[SNO 59] Snow C. P., The Two Cultures, Cambridge University Press, Londres, 1959. 
[TOF 17] TOFFIN G., « La fabrique de l'imaginaire », L’Homme, n²21, p. 167-190, 2017.

[VIN 88] VINCK D., «Imaginaires et biotechnologies », in Le triomphe des biotechnologies : la domestication de l'animal humain, Presse Universitaire de Namur, Namur, 1988. 